\title{
COMMENTS ON THE POWER OF KOLMOGOROV TEST
}

This paper presents the results of estimating the power of Kolmogorov test and its modifications for testing hypothesis about an exponential distribution against the alternative hypothesis (population has a Weibull distribution). The power of test was calculated based on a distribution of empirical cumulative distribution function and limiting distribution, as well as computer-simulation methods. Some important properties and limitations were given that are related to power simulation study of goodness-of-fit tests.

Keywords: Statistical test, Kolmogorov test, power of a test, exponential distribution, simulation.

\section{Introduction}

The $\lambda$ Kolmogorov test is one of the best-known goodness-offit tests for testing the hypothesis about the type of a probability distribution for a continuous distribution. Despite the vast literature, its use still arouses controversy. Among the most frequently tested hypotheses about the type of a distribution are the hypothesis of normality, also called normality test, and the hypothesis of exponentiality, or exponentiality test. To verify hypotheses about a normal distribution researchers use primarily tests (particularly for small samples) specialized in these types of hypotheses, called normality tests (e.g. Shapiro-Wilk, Epps-Pulley, skewness and kurtosis tests). Nonetheless, Kolmogorov test is still applied, as well as its modifications for testing normality of distribution, such as Kolmogorov-Lilliefors and other general goodness-of-fit tests, like Cramer-von Mises, Watson, Anderson-Darling tests. The importance of testing hypotheses about normal and exponential distributions is undeniable since such distributions play an important role in contemporary science and technology. It might be said that based on normal distribution are among others: statistics, metrology and prediction theory. Also, we cannot overate the importance of exponential distribution, especially in the theory of reliability and safety, diagnostics and maintenance theory, queuing theory and in various transport applications. The exponential distribution fields gives an important advantage of relatively simple calculations, whereas in the case of "competitive" distributions calculations are bound to be more complicated. Often it is not possible to obtain analytical solutions and, therefore, simulation study remains as the last alternative. However, the use of an exponential distribution is always combined with adopting strong assumptions, whose truth is frequently put into question. Thus, "the way" of assessing the admissibility of an exponential distribution is to verify the hypothesis based on empirical study that the probability distribution of the investigated variable is exponential, i.e. it does not differ in terms of statistical significance from exponential distribution. As long as this study is to provide reliable assessment of the applicability of exponential distribution, it is necessary to know what risk carries not rejecting a false hypothesis about an exponential distribution, i.e. what is the power of the used test. The vast majority of tests allow to control the type I error, i.e. the probability of rejecting a true hypothesis (so-called test of significance), however, most of them do not allow to control the error of type II, i.e. not rejecting a false hypothesis in favor of the alternative hypothesis. It has only been postulated to select tests (as well as size of random sample) so that the type II error would not exceed the type I error. Currently, it is rather postulated that an error of type II should not exceed 0.2 , thereby the power of a test should not be less than 0.8 $(80 \%)$. The issue of the test power creates another problem, namely, what decision to make when different tests give contradicting results.

In paper [1] we have analyzed the results compliance of testing the composite hypothesis of normality for 10 basic normality tests, including modified tests for composite hypotheses. The level of significance is equal to 0.05 . Simple samples of size up to $n=44$ were generated, coming from populations with either normal distribution or other distributions, like T-Student, Gamma, chi-square, exponential. It was found that at least eight tests gave the same result, approximately:

$85 \%$ when a sample was drawn from a normal distribution, $72 \%$ when a sample was drawn from a Student-t distribution, $70 \%$ when a sample was drawn from a chi-square distribution, $82 \%$ when a sample was drawn from a Gamma distribution, $100 \%$ when a sample was drawn from an exponential distribution.

However, only from 51 to $72 \%$ of choices for individual tests were correct, provided that excluded were the samples with an exponential distribution strongly differing from a normal distribution. The highest percentage of correct choices was observed for the skewness test (72\%), then for the Epps-Pulley test (67\%). The Shapiro-Wilk and Andreson-Darling test gave about $64 \%$ correct

\footnotetext{
* Andrzej Rogowski

Faculty of Transport and Electrical Engineering, University of Technology and Humanities, Radom, Poland,

E-mail: androgow@wp.pl; a.rogowski@uthrad.pl
} 
answers each, the classical Kolmogorov and Kolmogorov-Liliefors about $59 \%$, but the worst results were obtained for the Watson test (51\%) and Cramer-von Mises test (54\%).

Furthermore, a study was performed on testing hypotheses about an exponential distribution [2], including checking how many times individual tests do not give a reason to reject the hypothesis about an exponential distribution (composite hypothesis), whenever the generated sample did not come from a population with an exponential distribution (but with e.g. Gamma, power, Weibull, Erlang, chi-square). The verification was carried out for 20000 random samples. It turned out that the classical Kolmogorov test, for samples of size $n=25,50,75,100$ gave no reason to reject the hypothesis about an exponential distribution (so the false hypothesis) in $63 \%, 44 \%, 35 \%, 29 \%$ of cases respectively. The best results were obtained for the modified Andreson-Darling and KolmogorovStephens tests $(36 \%, 26 \%, 21 \%, 19 \%)$.

\section{Calculating the power of test}

The power of a statistical test depends significantly on the test construction, size of a random sample, null hypothesis and alternative hypothesis. In case of parametric tests, there is a bulk of literature on power analysis, as well as modules designed for analyzing the power of tests are available in statistical software. The situation is more difficult in the case of non-parametric tests that are goodness-of-fit tests assessing empirical distributions against hypothetical distributions, especially for composite hypotheses. Basically, the task is solvable if the form of cumulative distribution function (referred to as c.d.f. henceforth) is unambiguously specified in the alternative hypothesis. One of available methods is a method of computer simulation, i.e. determining a null hypothesis and generating random samples with a given distribution. Subsequently one should analyze the value of test statistics in relation to the critical value, as well as the value of test statistics with the replacement of null by alternative hypotheses. The fundamental theorem used in generating random samples with a given distribution is as follows:

Theorem 1 ([3]).

If $\mathrm{F}$ is the c.d.f. of a random variable $X$, the random variable $F(X)$ has a uniform distribution on the interval [0.1].

By generating a random sample $\left\{y_{i}\right\}$ with a uniform distribution on the interval (0.1), and then using the inverse function of the fixed c.d.f. of $F$, we obtain a random sample $\left\{x_{i}\right\}\left\{F^{-1}\left(y_{i}\right)\right\}$ of the desired probability distribution.

In our case, we are interested in the power of goodness-of-fit tests under the null hypothesis $H_{0}$ : the sample comes from a population with exponential distribution, against the alternative hypothesis $H_{1}$ : the sample comes from a population with A distribution, where $A$ is the Gamma, Weibull or power distribution. We will assume that empirical distribution is defined by empirical c.d.f:

$$
F_{n}(x)=\frac{1}{2} \operatorname{card}\left\{x_{i}: x_{i}<x\right\}
$$

While generating random samples and testing hypotheses, special attention should be given to certain restrictions and properties arising from chosen techniques of generating samples, types of distributions, as well as the form of hypothesis $H_{0}$ and used statistical tests.

\section{Gamma distribution}

A random variable $X$ has a gamma distribution with parameters $b, p$ (which is written $\Gamma(b, p)$ ), if the probability density distribution $f(x)$ is defined by the following formula:

$$
f(x)=\left\{\begin{array}{cl}
0 & , \quad x \leq 0 \\
\frac{b^{p} x^{p-1}}{\Gamma(p)} e^{-b x}, & x>0, b>0, p>0
\end{array}\right.
$$

where $b$ is called a rate parameter, $p$ is called a shape parameter ${ }^{1)}$ $\Gamma(p)=\int_{0}^{+\infty} x^{p-1} e^{-x} d x, p>0$ denotes the Euler's Gamma function. The c.d.f. of distribution is given by:

$$
F(x)=\left\{\begin{array}{cl}
0, & x \leq 0 \\
\frac{\Gamma(p, b x)}{\Gamma(p)}, & x>0, b>0, p>0
\end{array}\right.
$$

where $\Gamma(p, b x)=\int_{0}^{b x} t^{p-1} e^{-t} d t$ is called an incomplete Gamma function.

If a sample is generated from a population with a Gamma distribution, and subsequently with the use of the Kolmogorov, Kolmogorov-Stephens, Cramer-von Mises, Watson and Anderson-Darling tests a composite hypothesis is verified that a sample comes from a population with an exponential distribution, then the test result depends only on a shape parameter $p$ and the value of generated numbers from the interval $(0.1)$. Therefore, special attention should be put on the quality of the random number generator with a uniform distribution on the interval (0.1). However, the result does not depend on a rate parameter, i.e. on a fixed sequence $\left\{y_{i}\right\}$ of numbers from the interval $(0.1)$ and the fixed value of shape parameter $p$. The value of test statistics for each test is the same for all permissible values of a rate parameter $b$ (but, of course, it varies for different statistics). The reason behind this is that with a fixed size of random sample, statistical values depend only on the value of c.d.f. $G(x)$ with an exponential distribution for arguments $x_{i}=F^{-1}\left(y_{i}\right)$. As we show below, for a composite hypothesis the value of $G(x)$ does not depend on the rate parameter $\mathrm{b}$.

\section{Proof:}

Let $\left\{y_{i}\right\}, i=1, \ldots, n$, be a sequence of numbers from the interval $(0.1),\left\{z_{i}\right\}$ be a sequence of numbers with the property that for every $i=1, \ldots, n$ there

\footnotetext{
${ }^{1)}$ If $X$ has a distribution $\Gamma(b, p)$, then the random variable $Y=b X$ has a distribution $\Gamma(1, p)$. Background information on the use of probability distributions, statistical tests, and critical values can also be found in [6].
} 


$$
y_{i}=\frac{1}{\Gamma(p)} \int_{0}^{z_{i}} t^{p-1} e^{-t} d t
$$

Let $z_{i}=b x_{i}(b>0)$. According to (3) we have

$$
F\left(x_{i}\right)=\frac{1}{\Gamma(p)} \int_{0}^{b x_{i}=z_{i}} t^{p-1} e^{-t} d t=y_{i}
$$

and values $x_{i}=\frac{z_{i}}{b}$ come from a population with distribution $\Gamma(b, p)$.

We have:

$$
\bar{x}=\frac{1}{n} \sum_{i=1}^{n} x_{i}=\frac{1}{n} \sum_{i=1}^{n} \frac{z_{i}}{b}=\frac{1}{n b} \sum_{i=1}^{n} z_{i}=\frac{1}{b} \bar{z}
$$

and for the composite hypothesis that the sample comes from a population

with an exponential distribution we have:

$$
G\left(x_{i}\right)=1-e^{-\frac{x_{i}}{\bar{x}}}=1-\exp \left(-\frac{\frac{z_{i}}{b}}{\frac{1}{n b} \sum_{i=1}^{n} z_{i}}\right)=1-e^{-\frac{z_{i}}{\bar{z}}}
$$

So from (4), with fixed values of $p$ and $y_{i}$, the values of $z_{i}$ are unambiguously determined and the value of the c.d.f. $G\left(x_{i}\right)$ does not depend on the value of $b$.

\section{Power distribution}

A similar situation occurs in the case of a power distribution defined by the $c . d . f$ :

$$
F(x)=\left\{\begin{array}{cl}
0, & x \leq 0 \\
\left(\frac{x}{b}\right)^{\delta} & , x \in(0, b], b, \delta>0 \\
1, & x>b
\end{array}\right.
$$

where $b$ is a rate parameter and $\delta$ is a shape parameter.

$$
\begin{aligned}
& \text { If } y_{i}=\left(\frac{x_{i}}{b}\right)^{\delta} \text {, then } x_{i}=b\left(y_{i}\right)^{\frac{1}{\delta}}, \bar{x}=\frac{b}{n} \sum_{i=1}^{n}\left(y_{i}\right)^{\frac{1}{\delta}}, \\
& G\left(x_{i}\right)=1-e^{-\frac{x_{i}}{\bar{x}}}=1-\exp \left(-\frac{b\left(y_{i}\right)^{\frac{1}{\delta}}}{\frac{b}{n} \sum_{i=1}^{n}\left(y_{i}\right)^{\frac{1}{\delta}}}\right)= \\
& =1-\exp \left(-\frac{n\left(y_{i}\right)^{\frac{1}{\delta}}}{\sum_{i=1}^{n}\left(y_{i}\right)^{\frac{1}{\delta}}}\right)
\end{aligned}
$$

and with a fixed sequence $\left[y_{i}\right\}$ and the fixed value of shape parameter $\delta$, the value of the c.d.f. $G\left(x_{i}\right)$ does not depend on the rate parameter $b$.

\section{Weibull distribution}

If we generate a Weibull distribution defined by the c.d.f:

$$
F(x)=\left\{\begin{array}{cl}
0 & , \quad x \leq 0 \\
1-e^{-(b x)^{v}}, & x, b, v>0
\end{array}\right.
$$

where $v$ is a shape parameter, $b$ is a rate parameter, then:

$$
\begin{aligned}
& \text { If } y_{i}=e^{-\left(b x_{i}\right)^{v}} \text {, then } x_{i}=b^{-1}\left(-\ln y_{i}\right)^{\frac{1}{v}}, \\
& \bar{x}=\frac{1}{n b} \sum_{i=1}^{n}\left(-\ln y_{i}\right)^{\frac{1}{v}}, \\
& G\left(x_{i}\right)=1-e^{-\frac{x_{i}}{\bar{x}}}=1-\exp \left(-\frac{b^{-1}\left(-\ln y_{i}\right)^{\frac{1}{v}}}{\frac{1}{n b} \sum_{i=1}^{n}\left(-\ln y_{i}\right)^{\frac{1}{v}}}\right)= \\
& =1-\exp \left(-\frac{n\left(-\ln y_{i}\right)^{\frac{1}{v}}}{\sum_{i=1}^{n}\left(-\ln y_{i}\right)^{\frac{1}{v}}}\right)
\end{aligned}
$$

and with fixed sequence $\left\{y_{i}\right\}$ and the fixed value of shape parameter $v$, the value of the c.d.f. $G\left(x_{i}\right)$ does not depend on the rate parameter $b$.

If we generate a Weibull distribution with a fixed shape parameter $v$ and we form hypothesis $H_{0}$ that the distribution comes from a population with a Weibull distribution and with the same shape parameter $v$, then we use the property that if a random variable $X$ has a Weibull distribution with parameters $v$ and $b$, then a random variable $Z=X^{v}$ has an exponential distribution with parameter $b^{v}$ [4]. This means that the verification of the hypothesis that $X$ has a Weibull distribution, when a shape parameter $v$ is fixed, is equivalent to verifying the hypothesis that $Z$ has an exponential distribution. By using the previous calculations (12), (13):

$$
\begin{aligned}
& z_{i}=\left(x_{i}\right)^{v}=\left(b^{-1}\left(-\ln y_{i}\right)^{\frac{1}{v}}\right)^{v}=b^{-v}\left(-\ln y_{i}\right), \\
& \bar{z}=\frac{1}{n b^{v}} \sum_{i=1}^{n}\left(-\ln y_{i}\right) \\
& G\left(z_{i}\right)=1-e^{-\frac{z_{i}}{\bar{z}}}=1-\exp \left(-\frac{b^{-v}\left(\ln y_{i}\right)}{\frac{1}{n b^{v}} \sum_{i=1}^{n}\left(\ln y_{i}\right)}\right)= \\
& =1-\exp \left(-\frac{n \ln y_{i}}{\sum_{i=1}^{n} \ln y_{i}}\right)
\end{aligned}
$$

and with a fixed sequence $\left\{y_{i}\right]$ and the fixed value of shape parameter $v$, we found that the value of the c.d.f. $G\left(z_{i}\right)$ does not depend on the rate parameter $b$.

Note also that if for the fixed sequence $\left\{y_{i}\right\}$ we generate the exponential distribution (with parameter $\lambda=b$ ) described by the function:

$$
G(x)=\left\{\begin{array}{cl}
0 & , \quad x \leq 0 \\
1-e^{-\lambda x} & , \quad x>0, \lambda>0
\end{array}\right.
$$

we get a simple sample $\left\{x_{i}\right]$, then by assuming that $v=1$ (Weibull distribution for $v=1$ is exponential) and using (12) and (13) we obtain the same result as in (15), i.e. $G\left(x_{i}\right)=G\left(z_{i}\right)$, so the value of test statistics mentioned at the beginning of goodness-of-fit tests are the same. 
These results are important for examining the power of tests and the compatibility of test results, while verifying a hypothesis about an exponential distribution by means of a simulation method. With a fixed sample $\left\{y_{i}\right]$ drawn from a population with a uniform distribution from the interval (0.1), the result of goodness-of-fit test (for a composite hypothesis) does not depend on the rate parameter $b$ of generated distribution. This means that a rate parameter may be chosen freely, e.g. $b=1$, without affecting the results of simulation.

Using the above proven properties and results given in Fisz's book [3], we are able to calculate the power of tests based on the Kolmogorov's statistics for hypothesis about an exponential distribution against the alternative hypothesis (a sample comes from a population with Weibull distribution). For calculating can be used the values of c.d.f. of exponential and Weibull distributions (for a fixed shape parameter $p$ ) with the rate parameter $b=1$. Using the limiting distribution Fisz gives a lower bound of the $M$ power of $\lambda$ Kolmogorov test [3, p. 593] as:

$$
M \geq 1-\frac{1}{\sqrt{2 \pi}} \int_{a}^{b} e^{-\frac{z^{2}}{2}} d t
$$

where

$-\alpha=\frac{-\lambda_{\alpha}-\delta \sqrt{n}}{\sqrt{F\left(x_{0}\right)\left(1-F\left(x_{0}\right)\right)}}$

$-b=\frac{\lambda_{\alpha}-\delta \sqrt{n}}{\sqrt{F\left(x_{0}\right)\left(1-F\left(x_{0}\right)\right)}}$

- $\delta=\left|F\left(x_{0}\right)-G\left(x_{0}\right)\right|=\sup _{-\infty \leq v \leq+\infty}|F(x)-G(x)|$,

- $G(x)$ and $F(x)$ denote the $c . d . f$. of continuous distributions specified for null and alternative hypothesis respectively,

- $\lambda_{a}$ - critical value of test,

- $n$ - sample size.

At the same time

$$
\begin{aligned}
& M \geq 1-P\left(n G\left(x_{0}\right)-\lambda_{\alpha} \sqrt{n}<n S_{n}\left(x_{0}\right)<\right. \\
& \left.<n G\left(x_{0}\right)+\lambda_{\alpha} \sqrt{n}\right)
\end{aligned}
$$

where $S_{n}(x)=1 / n$ card $\left\{x_{i}: x_{i}<x\right\}$ is the empirical c.d.f. Because $n S_{n}(x)$, when the sample $\left\{x_{i}\right]$ comes from a population with distribution described by the c.d.f. $H(x)$, has a Bernoulli distribution with parameters $H(x)$ and $n$, so on the condition that the alternative hypothesis is true, the power of Kolmogorov test should not be less than the probability of getting at most $\left[n G\left(x_{0}\right)-\lambda_{a} \sqrt{n}\right]$ or at least $\left[1+n G\left(x_{0}\right)+\lambda_{a} \sqrt{n}\right]$ successes $^{2)}$ in $n$ Bernoulli's trials with probability of success $p=F\left(x_{0}\right)$.

\section{Calculating results}

Table 2 demonstrates the estimates of the power of classical $\lambda$ Kolmogorov and Kolmogorov-Stephens tests (Table 1) using the inequality (17) for a sample of size 100 . It was assumed that $H_{0}$ denotes the hypothesis that distribution in the general population is exponential distribution, and that $H_{1}$ denotes the alternative hypothesis that distribution in the general population is the Weibull distribution with a shape parameter $v$ (for selected values of the parameter $v$ ). The level of significance is equal to 0.05 . The rate parameter was assumed to be equal to 1 . Numerically were determined the values of exponential c.d.f. and Weibull distributions, the maximum difference between the $c . d . f$., as well as and the value of the argument, for which the difference reaches a maximum. Table 3 shows the estimates of test power obtained using the inequality (18) for a sample of size $n=25 ; 50 ; 75 ; 100 ; 1000 ; 10000$. Table 4 gives the estimates of test power collected by simulation study based on 10000 random samples. All calculations were performed in the Excel spreadsheet.

\section{Conclusions}

The analysis of Tables 2 and 3 shows that the lower estimates for both methods $(n=100)$ are very similar, so the differences might be considered negligible. At the same time the power of KS test is greater than of $\mathrm{K}$ test and the power of $\mathrm{K}-\mathrm{S} 1$ is greater than KS test. This is particularly evident for the value of rate parameter close to 1 . The differences diminish with the increase and decrease in the value of rate parameter, which is natural since then the power of test increases to 1 , and 1 is the upper bound of the power of test. Simulation study did not fully confirm estimates in Tables 2 and 3. The power of tests estimated on the basis of simulations is up to 10 times higher than what the formulas (17) and (18) indicate (it should be borne in mind that these are lower estimates). However, the nature of the changes of the test power is preserved, together with the change of the shape, as well as relationships between the individual tests. Tables 2, 3 and 4 show that the attempt to distinguish the exponential distribution from the Weibull distribution for shape parameter close to 1 is pointless for random samplings of a small size. Significant distinction occurs only for samples of a size approximately tens of thousands (according to Tables 2 and 3), but taking a sample of such size is virtually impossible. Simulation results suggest that at least for the KS test, and the K-S1 test that the sample size should be of at least a few thousand. However, samples of such size happen very rarely in practice. The results of Tables 2, 3 and 4 confirm the previously reached conclusion that the classical Kolmogorov test is not very useful at least for small and medium-sized samples and composite hypothesis. Therefore, the results of hypotheses verification with the use of this test must be approached with extreme caution.

2) where $n G\left(x_{0}\right)+\lambda_{a} \sqrt{n}$ is a natural number 


\begin{tabular}{|c|c|c|}
\hline Name and designation of the test & Test statistics & $\begin{array}{c}\text { Critical value of the test for the } \\
\text { significance level } \alpha=0.05\end{array}$ \\
\hline $\begin{array}{c}\text { Kolmogorov } \\
\mathbf{K}\end{array}$ & $D=\sqrt{n}\left(\max _{1 \leq i \leq n}\left(\left|\frac{i}{n}-F\left(x_{i}\right)\right|, F\left(x_{i}-\frac{i-1}{n}\right)\right)\right)$ & 1.3403 \\
\hline $\begin{array}{c}\text { Kolmogorov-Stephens } \\
\text { K-S }\end{array}$ & $D_{1}=\left(D+\frac{0.2}{n}\right)\left(\sqrt{n}+0.26+\frac{0.5}{\sqrt{n}}\right)$ & 1.094 \\
\hline $\begin{array}{c}\text { Kolmogorov-Stephens } \\
\text { K-S1 }\end{array}$ & $\sqrt{n} D$ & $n=100$ \\
\hline
\end{tabular}

Source: test and critical values of K - [4], K-S test, K-S1 and critical values - [5]

Estimates with the use of formula (17) of lower powers (in \%) of K, K-S and K-S1 tests for testing hypothesis about

Table 2 an exponential distribution and Weibull distribution as an alternative hypothesis for selected rate parameters $v$ for $n=100$

\begin{tabular}{|c|c|c|c|c|c|c|c|}
\hline \multirow{2}{*}{$v$} & \multirow{2}{*}{$x_{0}$} & \multirow{2}{*}{$G\left(x_{0}\right)$} & \multirow{2}{*}{$F\left(x_{0}\right)$} & \multirow{2}{*}{$\left|G\left(x_{0}\right)-F\left(x_{0}\right)\right|$} & \multicolumn{3}{|c|}{ M power of test [\%] } \\
\hline & & & & & K & K-S & K-S1 \\
\hline 0.30 & 0.101 & 0.3950960310044430 & 0.0960669671141359 & 0.2990290638903070 & 99.96 & 99.99 & 100.00 \\
\hline 0.40 & 0.129 & 0.3564748778867340 & 0.1210260344544170 & 0.2354488434323170 & 98.29 & 99.58 & 99.78 \\
\hline 0.50 & 0.155 & 0.3254438727669140 & 0.1435848225163860 & 0.1818590502505280 & 84.63 & 93.90 & 96.15 \\
\hline 0.60 & 0.179 & 0.2996777441522180 & 0.1638941006029640 & 0.1357836435492530 & 51.53 & 71.77 & 78.90 \\
\hline 0.65 & 0.190 & 0.28806863056666230 & 0.1730408660566380 & 0.1150277645099850 & 33.74 & 54.94 & 63.83 \\
\hline 0.70 & 0.201 & 0.2776640401393210 & 0.1820875684461410 & 0.0955764716931803 & 19.53 & 37.88 & 46.95 \\
\hline 0.75 & 0.212 & 0.2683327176459230 & 0.1910353024335000 & 0.0772974152124229 & 10.02 & 23.44 & 31.22 \\
\hline 0.80 & 0.222 & 0.2591618860026900 & 0.1990846356653410 & 0.0600772503373488 & 4.57 & 13.02 & 18.73 \\
\hline 0.85 & 0.232 & 0.2508716553216730 & 0.2070538766933160 & 0.0438177786283568 & 1.87 & 6.54 & 10.20 \\
\hline 0.90 & 0.241 & 0.2425919678739540 & 0.2141583736116540 & 0.0284335942622996 & 0.70 & 3.01 & 5.13 \\
\hline 0.95 & 0.250 & 0.2350488976032350 & 0.2211992169285950 & 0.0138496806746403 & 0.25 & 1.39 & 2.62 \\
\hline 0.98 & 0.256 & 0.2313137013105780 & 0.2258580312077520 & 0.0054556701028261 & 0.16 & 1.01 & 1.99 \\
\hline 1.02 & 0.263 & 0.2259104675072760 & 0.2312581026888400 & 0.0053476351815641 & 0.15 & 0.95 & 1.89 \\
\hline 1.05 & 0.268 & 0.2219181960499450 & 0.2350922188971360 & 0.0131740228471907 & 0.20 & 1.19 & 2.29 \\
\hline 1.10 & 0.276 & 0.2154625763106550 & 0.2411870692387590 & 0.0257244929281033 & 0.43 & 2.14 & 3.86 \\
\hline 1.15 & 0.284 & 0.2095353618438110 & 0.2472333552938040 & 0.0376979934499930 & 0.90 & 3.92 & 6.64 \\
\hline 1.20 & 0.292 & 0.2040950919851770 & 0.2532314640266430 & 0.0491363720414658 & 1.76 & 6.75 & 10.81 \\
\hline 1.30 & 0.307 & 0.1937947833844060 & 0.2643493990927470 & 0.0705546157083403 & 5.42 & 16.29 & 23.59 \\
\hline 1.40 & 0.322 & 0.1850623119905970 & 0.2753018096700970 & 0.0902394976794999 & 12.97 & 31.09 & 41.08 \\
\hline 1.50 & 0.335 & 0.1762558279746490 & 0.2846619136474400 & 0.1084060856727910 & 25.06 & 48.96 & 59.75 \\
\hline 1.70 & 0.360 & 0.1614505387433630 & 0.3023236739289690 & 0.1408731351856060 & 57.38 & 80.38 & 87.24 \\
\hline 2.00 & 0.394 & 0.1437869126506990 & 0.3256459437595560 & 0.1818590311088570 & 91.36 & 98.05 & 99.09 \\
\hline 2.50 & 0.441 & 0.1211584098674260 & 0.3566072934270440 & 0.2354488835596170 & 99.91 & 99.99 & 100.00 \\
\hline 3.00 & 0.480 & 0.1046960415637330 & 0.3812166081938590 & 0.2765205666301260 & 100.00 & 100.00 & 100.00 \\
\hline 4.00 & 0.541 & 0.0820957234975253 & 0.4178342046013590 & 0.3357384811038330 & 100.00 & 100.00 & 100.00 \\
\hline 5.00 & 0.588 & 0.0678754922000777 & 0.4445629513019820 & 0.3766874591019040 & 100.00 & 100.00 & 100.00 \\
\hline 10.00 & 0.718 & 0.0357570490498701 & 0.5122732653742690 & 0.4765162163243990 & 100.00 & 100.00 & 100.00 \\
\hline
\end{tabular}

Symbols as in formula (17) and in Table 1

Source: own elaboration 


\section{COMMNICOIIONS}

Estimates using the formula (18) of the power (in \%) of K, K-S and K-S1 tests for testing hypotheses of an exponential

Table 3 distribution and Weibull distribution as an alternative hypothesis for selected shape parameters $v$ and sample size $n$

\begin{tabular}{|c|c|c|c|c|c|c|c|c|c|c|c|c|c|c|c|c|c|c|c|c|c|}
\hline & \multicolumn{3}{|c|}{$n=25$} & \multicolumn{3}{|c|}{$n=50$} & \multicolumn{3}{|c|}{$n=75$} & \multicolumn{3}{|c|}{$n=100$} & \multicolumn{3}{|c|}{$n=1000$} & \multicolumn{3}{|c|}{$n=10000$} & \multicolumn{3}{|c|}{$n=100000$} \\
\hline & K & K-S & K-S1 & K & K-S & K-S1 & K & K-S & K-S1 & $\mathrm{K}$ & K-S & K-S1 & $\mathrm{K}$ & K-S & K-S1 & $\mathrm{K}$ & K-S & K-S1 & $\mathrm{K}$ & K-S & K-S \\
\hline & .51 & & & 30 & 93 & 93 & & & & & & & & & & & & & 00 & & \\
\hline & & & & & & & & & & & & & & & & & & & & & \\
\hline & 77 & .28 & & & .45 & .75 & & & & & & 63 & 0 & 100 & & & & & 00 & in & 00 \\
\hline & 14 & 85 & .85 & 03 & 14 & 32 & 67 & .78 & .78 & 40 & 23 & 65 & 00 & 100 & & & 00 & 100 & 00 & 00 & 00 \\
\hline 65 & 87 & 5.39 & 16.76 & 14 & 21.48 & 4.62 & 2.91 & 5.51 & 57.60 & 32.50 & .21 & 63.38 & 100 & 100 & 100 & 100 & 100 & 100 & 100 & 00 & 10 \\
\hline 70 & & 32 & 1.10 & 69 & 17.05 & 7.05 & .28 & .57 & 37.56 & 16.86 & .73 & .78 & 100 & 100 & 100 & 100 & 100 & 100 & 100 & 00 & 0 \\
\hline & & 45 & 47 & & .51 & 3.38 & 69 & 1.82 & .58 & & 3.07 & .88 & & 99.96 & & 100 & 100 & 100 & 100 & 00 & \\
\hline & 01 & 284 & 284 & & 4.97 & 4.99 & 109 & 22 & 9.60 & 66 & 8.38 & 19.86 & 1.50 & 97.67 & 98.68 & 00 & 100 & 100 & 00 & 00 & 0 \\
\hline 85 & 01 & 0.36 & 2.33 & 41 & 1.41 & 3.80 & 0.71 & 3.70 & 7.11 & 0.81 & 5.81 & 9.65 & 4.79 & 77.06 & 83.44 & 100 & 100 & 100 & 100 & 100 & 10 \\
\hline & 02 & 32 & 2.17 & 07 & 1.12 & 2.98 & 0.17 & 1.25 & 2.79 & 0.22 & 2.25 & 4.17 & 12.88 & 30.58 & 42.16 & 99.98 & 100 & 100 & 100 & 100 & 100 \\
\hline & & & & & 90 & 1.10 & & & & & & 1.72 & 1.04 & 3.02 & & 3.40 & 10.10 & & 100 & & 10 \\
\hline 98 & 03 & 29 & 0.62 & 10 & 0.34 & 1.06 & 0.08 & 0.52 & 1.23 & 0.07 & 0.70 & 1.46 & 0.21 & 135 & & 2.59 & 9.36 & 14.60 & 急 & 3.44 & 96. \\
\hline 1.02 & 04 & 0.30 & 0.70 & .09 & 0.36 & 1.07 & 0.10 & 0.57 & 1.29 & 0.15 & 0.64 & 1.26 & 0.25 & 1.37 & 2.40 & 2.50 & 8.88 & 13.31 & 78.51 & 92.12 & $95 .($ \\
\hline 1.05 & 05 & 31 & 0.76 & 11 & 80 & 11 & 0.12 & 0.64 & 1.40 & 0.17 & 0.75 & 1.42 & 1.25 & 4.86 & 7.52 & 45.62 & 69.27 & 77.04 & 100 & 100 & 100 \\
\hline & & & & & & & & & & & & & 8.87 & 22.21 & 31.86 & 99.77 & 99.97 & [9.9 & & & \\
\hline 1.15 & & 98 & 1.06 & 55 & 1.24 & 2.63 & 0.52 & 1.96 & 3.53 & 0.79 & 3.89 & 6.15 & .13 & 54.81 & 66.10 & 100 & 100 & 180 & 100 & $0 \Omega$ & \\
\hline 1.20 & 38 & 19 & 1.26 & 0.73 & 3.26 & 3.30 & 1.43 & 4.53 & 7.48 & 1.99 & 5.23 & 8.03 & 65.98 & 84.10 & 88.87 & 100 & 100 & 100 & 100 & 100 & 100 \\
\hline 1.30 & 57 & 69 & 36 & .22 & 4.88 & 8.76 & 2.51 & 7.15 & 11.21 & 5.72 & 12.63 & 17.76 & 97.39 & 99.43 & 99.70 & 100 & 100 & 100 & 100 & 00 & 0 \\
\hline 1.40 & & & 0.10 & 3.81 & 11.99 & 12.00 & 6.78 & .95 & 22.78 & 13.36 & 25.01 & 32.48 & 99.90 & 100 & & & & & 100 & 00 & \\
\hline 1.50 & & & & .26 & 15.30 & 3.54 & 4.45 & 28.68 & 37.79 & & 40.36 & & & & & & & & & & \\
\hline 1.70 & & .24 & 19.66 & 14.89 & 32.92 & 32.92 & 1.86 & 51.08 & 61.01 & 47.11 & 64.23 & 72.08 & 100 & 100 & 100 & 100 & 100 & 100 & 100 & 100 & 100 \\
\hline 2.00 & & 69 & 7.58 & .12 & .66 & 58.66 & 8.46 & 76.21 & 83.30 & 80.63 & 90.39 & 93.65 & & & 100 & 100 & & 100 & 00 & 00 & 10 \\
\hline 2.50 & .08 & .65 & 5.08 & 4.81 & 83.72 & 83.72 & 89.85 & 96.22 & 97.89 & 97.41 & 99.15 & 59.J & 100 & 100 & 100 & 100 & 100 & 100 & 100 & 100 & 100 \\
\hline 3.00 & 02 & 9.84 & 65.88 & 5.04 & 4.99 & 94.99 & 97.48 & 99.31 & 99.67 & 99.80 & 99.96 & 99.98 & & & & & & 100 & 100 & 00 & 100 \\
\hline 4.00 & .53 & & & 6.86 & 9.32 & 99.32 & 99.90 & 99.98 & 99.99 & & 100 & & & & & & & & & 00 & \\
\hline 5.00 & 02 & 39 & 92.91 & 99.44 & 99.78 & 99.92 & 99.99 & 100 & 100 & 100 & 100 & 100 & 100 & 100 & 100 & 100 & 100 & 100 & 100 & 100 & 100 \\
\hline 10.0 & 5.83 & 98.40 & 99.48 & 9.99 & 100 & 100 & 100 & 100 & 100 & 100 & 100 & 100 & 100 & 100 & 100 & 100 & 100 & 100 & 100 & 100 & 10 \\
\hline
\end{tabular}

Symbols as in Table 2

Source: own elaboration

Estimates of power (in \%) of K, KS and K-S1 tests for testing hypotheses of an exponential and Weibull distributions

Table 4 for selected shape parameters, when samples came from Weibull distribution. Simulation study of 10000 random samples

\begin{tabular}{|c|c|c|c|}
\hline$v$ & K & K-S & K-S1 \\
\hline 0.85 & 14.05 & 42.26 & 48.63 \\
\hline 0.90 & 4.11 & 20.07 & 24.94 \\
\hline 0.95 & 1.35 & 9.51 & 13.04 \\
\hline 0.98 & 0.65 & 6.87 & 10.02 \\
\hline 1.02 & 0.58 & 7.71 & 10.45 \\
\hline 1.05 & 1.09 & 10.20 & 13.51 \\
\hline 1.10 & 3.68 & 19.91 & 25.15 \\
\hline 1.15 & 7.49 & 32.54 & 39.06 \\
\hline 1.20 & 14.46 & 48.21 & 55.59 \\
\hline
\end{tabular}

Symbols as in formula (17) and in Table 2

Source: own elaboration

$94 \cdot$ COMMUNICATIONS $3 / 2013$ 
Note that the statistical tests do not distinguish between an exponential distribution and other distributions, such as Weibull or gamma, as so for a particular group of parameters this can be an important argument for the application of an exponential dis- tribution in various models, despite theoretical objections to its use. If the differences are "invisible" to the statistical apparatus, they might be considered negligible.

\section{References}

[1] ROGOWSKA, R., ROGOWSKI, A.: Compliance of the Results of Hypothesis Testing with Normal Distribution for Selected Statistical Tests (in Polish), Logistyka, No. 3, 2011 (Logistyka - nauka, materialy VIII Konferencji Naukowo-Technicznej „Logitrans” - Logistyka, Systemy Transportowe, Bezpieczenstwo w Transporcie).

[2] ROGOWSKI, A.: Compliance of the Results of Hypothesis Testing with Exponential Distribution for Selected Statistical Tests, The Archives of Transport, vol. 24, No. 4, 2012.

[3] FISZ, M.: Probability Theory and Mathematical Statistics (in Polish), PWN, Warszawa 1967.

[4] FIRKOWICZ, S.: Statistical Testing of Products, WNT, Warszawa 1970.

[5] DOMANSKI, CZ:: Nonparametric Statistical Tests (in Polish), PWE, Warszawa 1979.

[6] ROGOWSKI, A.: The Basics of Probabilistic Methods in Transport (in Polish), seria Monografie, No. 175, Wydawnictwo Uniwersytetu Technologiczno-Humanistycznego, Radom 2012. 